\title{
The wobbly garden table
}

\section{Hanspeter Kraft}

Department of Mathematics, University of Basel, Rheinsprung 21, CH-4051 Basel, Switzerland

The situation is undoubtedly familiar: friends are sitting outside in the fresh air, gathered in the best of humour around a garden table awaiting some cool drinks. Unfortunately, the table is a bit wobbly and the drinks are slopping out of the glasses. The reaction is always the same: the table is pushed to and fro, with no result other than splashing even more out of the glasses, and then someone finds a bit of paper or cardboard and pushes it beneath whichever table leg is too short. This usually succeeds in at least reducing the wobble sufficiently to prevent the slopping, at any rate as long as no one bumps into the table and moves it again. Thus the matter is resolved, and the party can continue!

Here we want to use this familiar course of events in order to analyze our own behaviour in such situations. There is in fact a surprising and very elegant solution to the problem, as we shall shortly see. And, even more interesting than the solution itself is the question, how did one arrive at it? We shall attempt to imagine how an engineer, a physicist and a mathematician would tackle the problem.

\section{THE ENGINEER}

Let us first imagine how a typical engineer would approach such a problem. He establishes that one of the table legs is too short (or too long?), and that the wobble could therefore readily be eliminated if the lengths of the table legs were variable. A brief reflexion convinces him that it is sufficient if only one of the legs is adjustable. Of course, he immediately has an idea how one could actually construct such a leg-with the help of a threaded rod. But before he starts serious work, there are a few things to clear up, in particular:

Patents: Does anything similar exist already, and if not, would the invention be suitable for patenting?

Market situation: Is there a demonstrable need for such a product ("anti-wobble feet"), and if so, with which firms could one cooperate for its development?

If the answers are positive, a first prototype will be built and tested in the field. In the event of a successful outcome, the financing of the project must be clarified. Once that is done, things proceed in wellknown ways: either the patent is sold to a firm, or an investor is found and one produces the anti-wobble feet oneself. A central issue has still to be solved, namely how the product is to be sold, i.e. its worldwide marketing. In planning all this it must be borne in mind that everyone concerned expects a financial return from their efforts.

The approach of the engineer is essentially that of tackling the symptom, without reflecting at length on the origin of the problem. New knowledge is not required to tackle it; but on the other hand quite a few people are involved and eventually earn something. One could call the operation one of interdisciplinary technology transfer, which creates employment and increases the gross national product.

\section{THE PHYSICIST}

Let us next consider a typical experimenter, for example, a physicist. He establishes that related problems also occur elsewhere, and that some of them have indeed already been solved. For example, a car moves on four wheels and does not wobble when on uneven ground, nor is one of the wheels occasionally in the air, at least not when the car is driven normally!

The physicist forms the impression that the problem is indeed interesting, and that it is worth attempting to find a more exact solution. He therefore decides to submit a grant proposal entitled "On the stability of multiply-legged objects on rough surfaces", in which the problem is formulated generally, existing solutions are discussed, and new ideas and possible approaches are suggested.

As soon as the grant is awarded and the money has arrived, the research team is assembled and the experiments can start. Among other things, it is established that arbitrary displacements of the object hardly ever eliminates the wobble. This observation can be formulated as a theoretical proposition: namely, that the probability of finding such a stable position through arbitrary displacements is zero.

Then, the idea of "damped feet", in analogy to car springs and shock absorbers, is investigated. This, however, introduces the danger that the whole table begins to oscillate, a problem that certainly requires more detailed investigation.

Meanwhile time has passed and the first progress report is due. Usually it will end with the assertion that further funds are needed in order to bring the project to a successful conclusion. There is no need to enter into the details, which are well enough known 
to the initiated. The comprehensive experiments and experimental results are fully documented and complemented with statistical analyses. As a rule these form the basis for several undergraduate, and perhaps even doctoral theses. It is also to be expected that the results themselves lead to new ideas and hence further new projects.

Thus the physicist can amply demonstrate the relevance of his research to both the solution of practical problems and to the encouragement of the coming generation of scientists by giving them the possibility to distinguish themselves in research work. The activity thereby significantly contributes to the continuation of his discipline.

\section{THE MATHEMATICIAN}

Finally, let us consider a typical pure scientist, for example a mathematician.

He first poses the question, "What is wobbling?"

An important observation emerges from this, namely that wobbling implies that two opposite legs are touching the ground-the straight line passing through them is the wobble axis (figure 1a) - and one of the other two legs is in the air. A central discovery follows: There are two wobble states for a four legged table, corresponding to the two possible wobble axes. The mathematician then carries out the following thought experiment. He numbers the legs 1 to 4 and begins in the state corresponding to the wobble axis $1-3$, i.e. legs numbers 1 and 3 are touching the ground and the wobble axis runs through them, while either

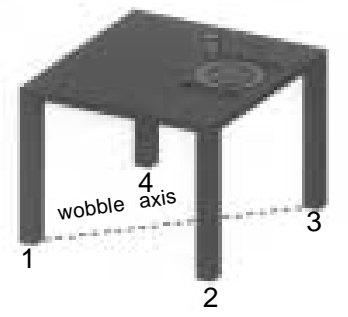

a

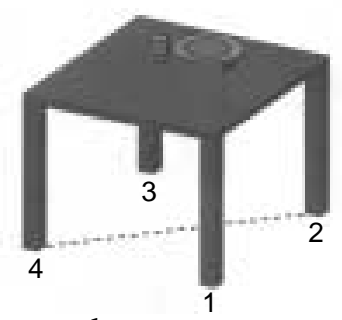

b
Figure 1. a, before; and b, after rotation. See text for explanation.

leg 2 or leg 4 is in the air. Now he lifts the table (in his imagination), turns it through $90^{\circ}$, and places it back onto the ground. Of course the table wobbles as before, since the ground itself has not changed (we assume that the table is correctly constructed, that is, it does not wobble on a flat surface, which is certainly a reasonable assumption in practice). From the viewpoint of the table, however, the situation has changed insofar as the wobble axis now runs through the legs numbered 2 and 4 ! - the table has been turned through $90^{\circ}$ (figure $1 \mathrm{~b}$ ). From this he can now draw the following conclusion: In the course of rotating the table by $90^{\circ}$ the original wobble axis switches to the other one, perpendicular to the original. There must therefore exist an intermediate position at which the transition between the two wobble axes takes place. In this position the table cannot wobble!

He has thereby established the following result: If one rotates a wobbly garden table about its own axis in an arbitrary direction, within $90^{\circ}$ of rotation one reaches a state in which the table no longer wobbles.

This surprisingly simple result cannot unfortunately be patented, i.e., there is no technology to be "transferred" or otherwise financially exploited. Even worse, it has not been achieved by interdisciplinary effort, nor has it led to any research projects, and neither undergraduate nor doctoral theses. It is, purely and simply, ingenious, elegant and effective! Incidentally, few people really believe that it works, for most lack confidence in abstract, logical conclusions, especially when they concern so-called practical problems.

We leave it to you to test the method in practice. You may thereby mightily impress your guests! If you care to add that you learned it from a (pure) mathematician, all mathematicians will be grateful to you.

In conclusion, you might note that this method is based on a result from calculus, namely the so-called Intermediate Value Theorem that states that a continuous function $f$ on the interval $[a, b]$ takes on each value between the two boundary values $f(a)$ and $f(b)$.

\section{ACKNOWLEDGMENTS}

This article first appeared in German in Uni Nova, a science magazine published by the University of Basel, in June 2000. I'm grateful to Jeremy Ramsden for the English translation, and to Lance Small for the fine tuning. 